\title{
Using Fuzzy Link Cost and Dynamic Choice of Link Quality Metrics to Achieve QoS and QoE in Wireless Mesh Networks
}

\author{
Rafael Lopes Gomes ${ }^{\mathrm{a}}$, Waldir Moreira Junior ${ }^{\mathrm{b}}$, Eduardo Cerqueira ${ }^{\mathrm{c}}$, \\ Antônio Jorge Abelém ${ }^{a}$ \\ ${ }^{a}$ Federal University of Para, Para, Brazil \\ ${ }^{b}$ Institute for Systems and Computer Engineering of Porto (INESC-Porto), Porto, \\ Portugal \\ ${ }^{c}$ Centre for Informatics and Systems of the University of Coimbra (CISUC), Coimbra, \\ Portugal
}

\begin{abstract}
The growth of multimedia applications and wireless systems requires a new behavior of routing protocols for Wireless Mesh Networks (WMNs). It is necessary to provide not only the minimum requirements for Quality of Service (QoS), but also to assure the Quality of Experience (QoE) support for multimedia applications. In this context, the usage of only one link quality metric for routing different types of packets within the network is not enough to ensure applications with suitable QoS and QoE levels. This paper presents a variation of the WMN routing protocol Optimized Link State Routing (OLSR), to achieve QoS and QoE requirements for multimedia applications. It is based on the dynamic choice of metrics and in a Fuzzy Link Cost (FLC) to determine the best routes for multimedia packets. The proposed FLC metric is based on a fuzzy system that uses two link quality metrics, namely Expected Transmission Count (ETX) and Minimum Delay (MD), to define a new metric. Simulations were performed to demonstrate the performance of the proposed metric compared to the ones present in the original OLSR and other current versions of this protocol. For comparison purposes, it was considered different performance evaluation QoS metrics and the quality of videos received by the user in a higher competition scenario.
\end{abstract}

Key words: Wireless Mesh Networks, QoS, QoE, Fuzzy Systems, Dynamic Choice of Link Quality Metrics

Email addresses: rafaellgom@gmail.com (Rafael Lopes Gomes), waldir.junior@inescporto.pt (Waldir Moreira Junior), cerqueira@ufpa.br (Eduardo Cerqueira), abelem@ufpa.br (Antônio Jorge Abelém ) 


\section{Introduction}

Wireless Mesh Networks (WMNs) are a special case of Ad hoc networks which allow multiple hops, and have low cost and ubiquitous features for Internet access and multimedia content distribution. A WMN consists of clients, routers, and gateways where routers provide connectivity to a set of fixed and/or mobile users and gateways provide connectivity to the Internet. In this scenario, an efficient and wise choice for communication routes becomes a major challenge for the success of the WMN [1].

Despite the constant evolution of wireless networks, they still have limited bandwidth, a large control packet overhead, and are strongly influenced by environmental factors such as weather, physical obstacles, interference, among others [8]. Due to these factors, the selection of routing protocols has great importance on the performance of the network as well as on the user perception.

Routing protocols for WMNs must follow the self-configuring, self-managing, and self-recovering principles as well as considering isotonic, traffic prediction, and overhead reduction issues [7]. Recently, several protocols were developed aiming to meet the different demands of specific multimedia applications since each application has its own characteristics. Among these demands stand out loss tolerance, minimizing end-to-end delay, and maximizing throughput.

The growth of the Internet, multimedia-based services and wireless access have motivated the development of applications and devices that use the resources of this "type" of Internet access. Thus, users expect to obtain the same types of services that are offered when they are in wired networks with at least the same quality level.

Within this scenario, WMNs should be designed to simultaneously transport data traffic and multimedia content with different QoS/QoE requirements to a large number of users. Hence, routing protocols must be studied, improved, and proposed to increase the performance of networks and the satisfaction of users.

By analyzing the problems related to QoS in WMN routing, we have the following observations: a good Internet service needs to consider a set of criteria (e.g., bit-error rate, bandwidth, delay, loss, and jitter) which depends on the situation (e.g., number of users, amount of traffic, noise and others) of 
the network at a given moment in order to attend the demands of multimedia applications (e.g., enough throughput, acceptable delay and loss, etc) and users (e.g., increase of experience, acceptable quality level and luminance).

In addition, to improve the performance of networks and to provide an efficient distribution of multimedia content, routing algorithms should also consider objective and subjective aspects regarding QoE and multimedia issues (e.g., blurring, noise and color distortion).

QoS metrics have been developed as a way of enabling traffic differentiation and allowing better quality services. Solutions based on QoS provide a set of control and assessment operations at the network (packet) level to ensure content dissemination with delivery guarantees.

Traditional QoS metrics, such as packet loss, delay, and jitter, are typically used to indicate the impact of network conditions on multimedia streams, but do not reflect the experience of end-users. Consequently, QoS parameters fail regarding the evaluation of the content quality from the user's perspective.

In order to fulfill the gaps related with QoS/QoE-awareness routing approaches, new schemes have been proposed [9][6]. QoE-based routing protocols aim to optimize the usage of network resources, the system performance and the quality level of multimedia applications. Therefore, with new WMN routing solutions, wireless operators can offer new services, reduce operational costs, while keeping and attracting new clients.

Thus, the scientific community along with industry have been working to propose scenarios, requirements, metrics and routing QoE mechanisms as a way to extend the traditional QoS models, optimizing the network resources and improving service and customer satisfaction. International Telecommunication Union - Telecommunication Standardization Sector (ITU-T) [10], Video Quality Experts Group (VQEG) [11], and European Technical Committee for Speech, Transmission, Planning, and Quality of Service (ETSI STQ) [12] are examples of organizations that invest in QoE as a way to assure suitable support for end-users.

In order to improve the distribution performance of multimedia content systems and increase user satisfaction in WMNs, this paper applies a fuzzy logic approach using a multiple-metric scheme with the Optimized Link State Routing (OLSR) [13] protocol. The proposed metric is defined through a fuzzy link cost for each link known in the network based on the crisp values of the different metrics used.

The fuzzy system creates a Fuzzy Link Cost (FLC) whose parameter val- 
ues are based on link quality metrics, namely Expected Transmission Count (ETX) [14] and Minimum Delay (MD) [15], which are collected from the network, allowing the use of this FLC value for routing multimedia-based User Datagram Protocol (UDP) packets. Transmission Control Protocol (TCP) packets continue to be routed according to the ETX metric, as in [16]. This proposed version of the OLSR protocol is named Optimized Link State Routing - Fuzzy Link Cost (OLSR-FLC).

OLSR-FLC is evaluated in a simulation scenario using Network Simulator 2 (NS-2) where real video transmissions are delivered and evaluated using objective and subjective QoE metrics in order to reflect the user experience. Additionally, QoS metrics are analyzed to verify the behavior of the proposed solution from the network point of view.

This paper is organized as following. Section 2 presents related work regarding fuzzy logic, routing and QoE/QoS. Section 3 introduces the OLSR protocol and a few popular extensions. Section 4 describes the proposed solution. Section 5 shows the performance evaluation. And, finally, Section 6 summarizes the paper and presents future work.

\section{Related Work}

This section presents existing works regarding the utilization of fuzzy logic in routing strategies and other proposals that use multiple metrics to provide QoS in wireless networks.

Aboelela and Douligeris [24] used a fuzzy logic approach to define a fuzzy cost to reflect the crisp values of the different metrics possibly used in the Broandband Integrated Services Digital Network (B-ISDN) links, integrating the fuzzy logic into the routing system. Thus, the throughput of the network was incresed.

Zhang and Klong [23] proposed a reflection about the necessity of multiple metrics to achieve QoS routing for the transmission and distribution of digitized audio/video across next-generation high-speed networks. Moreover, the authors introduced a fuzzy system to realize QoS routing with multiple metrics.

Lekcharoen et al [8] developed fuzzy control policing mechanisms to detect violations in parameter negotiation in wireless networks. Due to the demand for inexpensive but reliable models, the proposed fuzzy modeling approach turned out to be a useful complement to traditional modeling and control 
approaches when both the complexity and uncertainty about the system increases.

Moreira et all [18] proposes the use of multiple metrics with the proactive OLSR protocol, in order to provide quality of service routing in WMNs, emphasizing that it has already been proved that routing with multiple metrics is an NP-complete problem. The solution uses the techniques of Analytic Hierarchy Process (AHP) and Pruning combined to perform multiple metric routing for VoIP calls.

Regarding QoE schemes, existing works aim to provide intelligent packet dropper mechanisms [5], multimedia assessment solutions [4], QoE-aware handover controllers [3] and fairness-based resource allocation approaches for video applications [2], but QoE routing mechanisms are still a research goal and need to be developed to improve the usage of wireless resources and user satistaction.

Amongst the aforementioned papers, none proposes a protocol that aims to achieve QoS and QoE through the usage of dynamic choice of metrics and a multiple-metric approach based on fuzzy logic. We show that the protocol can wisely and efficiently optimize the network performance as well as improve the quality of the service given to the customer. Thus, it is expected that users feel encouraged to use WMNs for common internet multimedia services, making WMNs widely used for last-mile Internet access.

\section{OLSR Protocol}

The OLSR protocol [13] is an adaptation of the traditional link-state algorithm for Ad hoc networks. It is a proactive protocol which uses a routing table obtained through the exchange of messages between nodes about the network conditions.

An advantage of the OLSR protocol, from the QoS perspective, is its proactive nature that allows routes to be available even before the source needs to start a packet flow to a destination. Another advantage of the OLSR protocol, that uses link-state algorithm, is that route computation is performed using the knowledge about the entire network.

However, the hop count metric natively used by OLSR is unable to support QoS, since a selected route based on the lowest number of hops cannot satisfy the QoS requirements of multimedia packets that will be traversing the network. 
Thus, metrics which somehow represent the quality of links were proposed, and the ones of interest to this work are presented next.

\section{1. $O L S R-E T X$}

The OLSR extension based on ETX metric proposed in [14] aims to find routes with the lowest expected number of transmissions that are necessary to ensure that a package can be delivered and have its arrival confirmed by the final destination.

\section{2. $O L S R-M D$}

The main idea of OLSR Minimum Delay [15] is to measure the link delay, calculating it through the Ad hoc Probe technique. Therefore, the calculation of the routing table can be based on the delay calculated to each neighboring node. Hence, in the OLSR-MD protocol the route selection between the current node and any other node in the network will have as criteria the lowest sum of the different transmission delays of all links along the path.

\section{3. $O L S R-D C$}

The OLSR - Dynamic Choice (OLSR-DC) extension [16] aims to provide QoS support, giving different treatment to traffic from applications that use TCP and UDP, using the ETX metric [14] for routing TCP packets and MD metric [15] for routing UDP of packets. The protocol can also differentiate the routing of TCP and UDP packets, this is achieved due to each packet be routed according to the metrics that best reflect their needs.

This protocol is used as basis for this paper proposal, since the proposed FLC is based on metrics that express the characteristics relevant to multimedia traffic. And using the OLSR-DC as basis, we can use FLC to route only UDP packets usually used for multimedia applications.

Next, it is presented the OLSR-FLC along with the characteristcs of the fuzzy system developed and the main idea behind the usage of fuzzy logic to overcome the multiple metrics utilization.

\section{Proposed Extension: OLSR-FLC}

A multiple-metric routing approach is used to ensure that route selection comprises good quality links. With this goal, it is generated a metric based on a set of metrics. The result is a more complete metric since it will gather good 
characteristics of the ones considered for its development. Multiple-metric routing design is not trivial, and proved to be an NP-complete problem [17].

In this paper, the strategy used to overcome this problem was the fuzzy logic, proposed by Zadeh [21], because it has the advantage that the solution can be cast in terms of human perception. Therefore, such perception can be used in the design of the routing solution.

The idea of fuzzy sets is an extension of the traditional concept of sets (crisp) where one element belongs or not to a certain set. The fuzzy sets, in contrast, are defined from membership functions that are limited to an interval between 0 and 1, i.e., any value between 0 and 1 can express the membership degree of a certain element of the fuzzy set based on the inference functions used. Usually, the relevance degree of a value " $\mathrm{x}$ " regarding a function is represented by $\mu(\mathrm{x})$.

A fuzzy system has the following steps:

- Fuzzification: In this step, data regarding topology information is transformed into linguistic variables that are used in the available system of inference.

- Inference System or Inference Engine: the linguistic variables coming from the fuzzification process are applied to a specified set of rules and produce a set of linguistic variables related to the inference output.

- Defuzzification: uses the linguistic variables coming from the inference system and converts them into crisp values according to the defuzzification strategy being used.

The characteristics of the proposed fuzzy system are based on well-known heuristic evaluation done from membership functions, inference models, fuzzification and defuzzification methods found in the literature [22].

\subsection{Fuzzification}

The fuzzification process has as input the data received from the topology (i.e., ETX and MD values). Therefore, two membership functions are used, one for each metric. In the two functions, triangular and trapezoidal functions were used since, based on a heuristic evaluation executed during the development of the fuzzy system, they met the needs of the proposal regarding the others available in the literature, such as gaussian and sigmoidal 
functions that have a higher processing cost to calculate the relevance degree $[22]$.

A triangular function has three parameters: $a, b$ and $m$. Being "a" the first and " $\mathrm{b}$ " the last point where $\mu(\mathrm{x})$ is zero and " $\mathrm{m}$ " the point where $\mu(\mathrm{x})$ has value 1 . The relevance degree of a triangular function is determined by $[22]$ :

$$
\mu(x)=\left\{\begin{array}{c}
0 \text { if } x \leq a \\
(x-a) /(m-a) \text { if } x \in[a, m] \\
(b-x) /(b-m) \text { if } x \in[m, b] \\
0 \text { if } x \geq b
\end{array}\right.
$$

A trapezoidal function has four parameters: a, b, m1 and m2. Being "a" the first and "b" the last point where $\mu(\mathrm{x})$ is zero, and parameters " $\mathrm{m} 1$ " and " $\mathrm{m} 2$ " represent the range of points where $\mu(\mathrm{x})$ has value 1 . The relevance degree of a trapezoidal function is determined by [22]:

$$
\mu(x)=\left\{\begin{array}{c}
0 \text { if } x \leq a \\
(x-a) /(m-a) \text { if } x \epsilon[a, m 1] \\
1 \text { if } x \epsilon[m 1, m 2] \\
(b-x) /(b-m) \text { if } x \in[m 2, b] \\
0 \text { if } x \geq b
\end{array}\right.
$$

The membership function used for the received ETX values is shown in Figure 1 which has three linguistic variables, defined by the trapezoidal functions: high, medium, and low. The variables are organized as follows:

- High: Trapezoidal(x; 1, 1, 1.23, 1.56);

- Medium: Trapezoidal(x; 1.23, 1.56, 2.78, 4);

- Low: Trapezoidal(x; 2.78, 4, 100, 100).

The ETX metric calculation is expressed from an analysis of a 10-packet window through the formula ETX $=1 /\left(\mathrm{LQ}^{*} \mathrm{NLQ}\right)$, where LQ and NLQ are the quality of the link towards a neighbor and the link quality of the neighbor towards the actual node, respectively.

Thus, according to the defined function, a link is considered completely "high" when, in both directions, it loses at most one package, i.e., it has the ETX value between 1 and 1.23. The same idea works for the other 


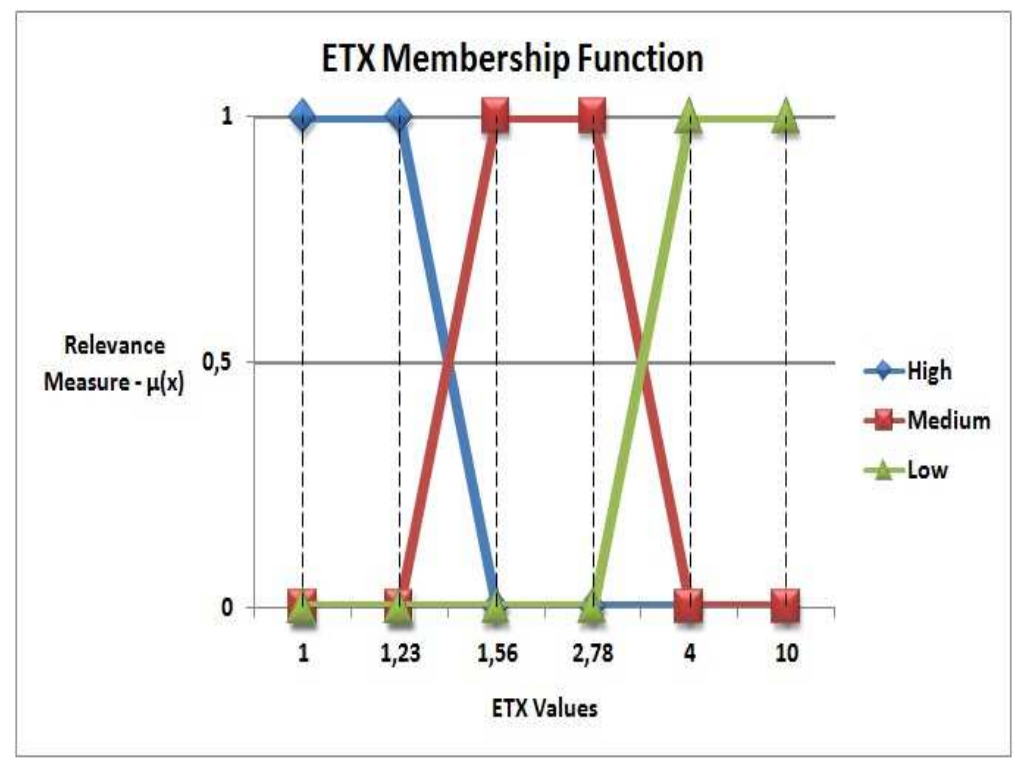

Figure 1: ETX Input Membership Function.

two functions, one link is considered completely "Medium" if its losses are between 2 and 4 packets. A link is considered "Low" when it has lost 5 or more packets.

The membership function used for the received MD values is shown in Figure 2 which has three linguistic variables, defined by two trapezoidal functions and one triangular function: high, medium, and low. The variables are organized as follows:

- High: trapezoidal(x; 0, 0, 1, 3);

- Medium: triangular(x; $1,3,5)$;

- Low: trapezoidal(x; 3, 5, 10, 10);

To select the parameters of the MD membership function was carried out a study to know how the propagation delay (measured by the MD metric) can add to the end-to-end delay in a multimedia flow. This study used one VoIP call in a scenario with only two nodes, i.e., one hop scenario. The end-to-end delay was related with the propagation delay, so we could view a relation between them. 


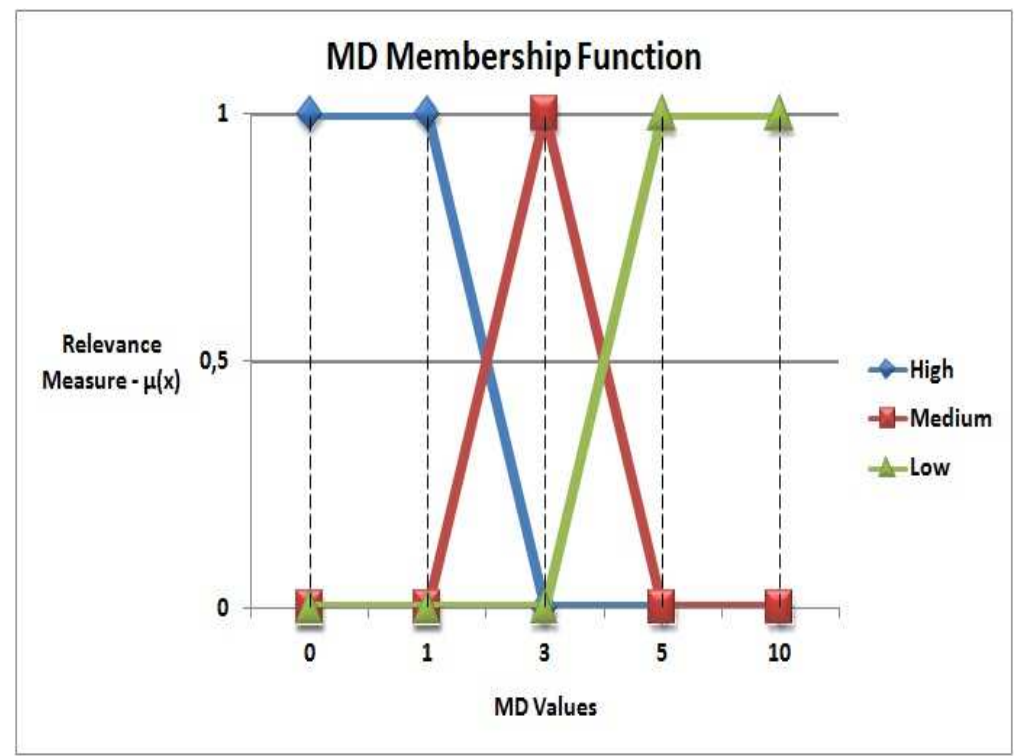

Figure 2: MD Input Membership Function.

This relation shows that delays up to one millisecond, give a lower influence in the end-to-end delay, in the same way, we checked that values between two and four milliseconds result in a small impact in the end-to-end delay. Finally, values after five milliseconds cause a greater influence in the end-to-end delay. Based on this study, we decided to build the membership function showed in Figure 2.

A WMN backbone is, in general, composed of routers with limited capacity of processing and memory. Thus, we decided to develop a fuzzy system that uses low requirements of the existing resource-constrained routers. Therefore, the proposal uses only functions that have a low cost of memory and processing. Thus, we can improve the overall WMNs performance, keeping the system scalability which is one of the main features of the WMNs. From the two membership functions shown, we obtain the linguistic variables used in the inference system which is described in the next sections.

\subsection{Inference System}

The inference system uses the membership function of output shown in Figure 3, where the possible values of FLC and their relevance degrees are expressed. The variables are organized as follows:

- High: triangular(x; 1, 1, 2); 
- Medium: triangular(x; 1, 2, 4);

- Low: triangular(x; 2, 4, 4);

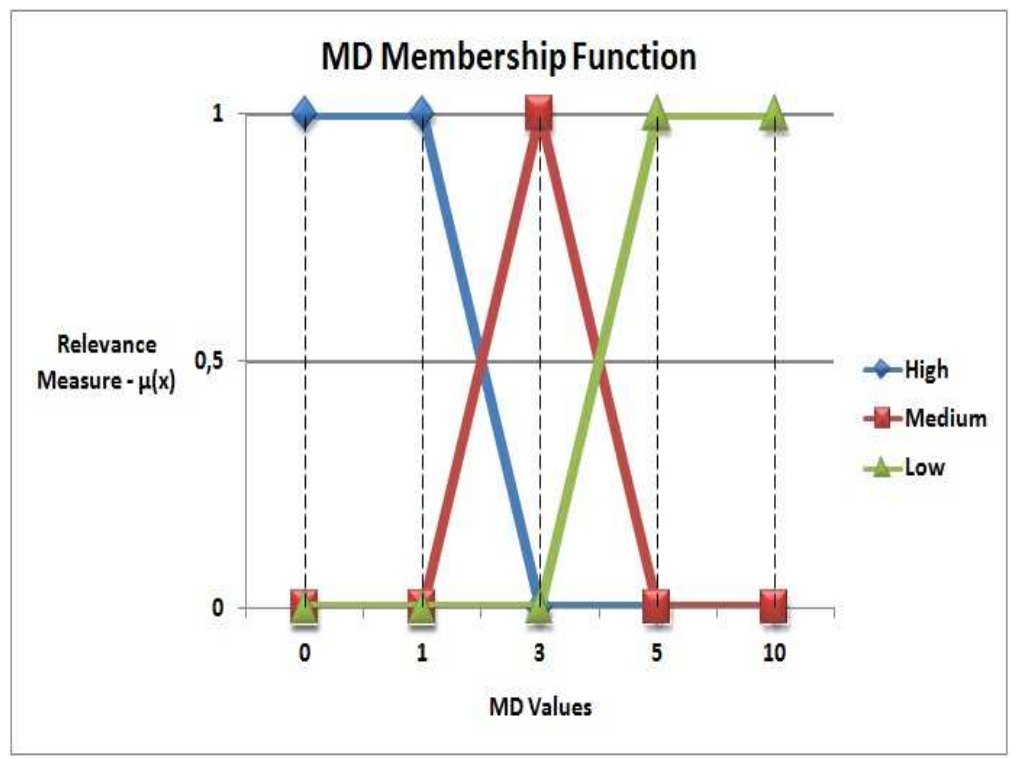

Figure 3: FLC Input Membership Function.

The distribution of linguistic variables aims to make a link considered completely "Medium" to have a doubled cost of a link which is considered completely "High". The same idea is applied to the links considered completely "Low" and "Medium".

This distribution results in the usage of a greater number of hops when the links with less number of hops are considered bad. This decreases the constant exchange of routes to be used at the time, consequently, a route is changed only when it becomes bad when compared to a newly discovered route.

The inference system uses a set of rules presented in Table 1, which expresses the possible output linguistic variables according to the input linguistic variables coming from the fuzzification process.

The operator "or" used in Rule 5 of Table 1 represents a union operation of two fuzzy sets which can be represented by the function proposed by Zadeh [21]: $\mu \mathrm{A} \cup \mathrm{B}=\operatorname{Max}[\mu \mathrm{A}(\mathrm{xi}), \mu \mathrm{B}(\mathrm{xi})]$. The operator "and" represents the 
Table 1: Fuzzy Rules

\begin{tabular}{|c|c|c|c|c|}
\hline Rules & ETX & Operation & MD & Fuzzy Link Cost (FLC) \\
\hline 1 & High & And & High & High \\
\hline 2 & High & And & Medium & Medium \\
\hline 3 & Medium & And & High & Medium \\
\hline 4 & Medium & And & Medium & Medium \\
\hline 5 & Low & Or & Low & High \\
\hline
\end{tabular}

intersection between two fuzzy sets which can be represented by the function also proposed by Zadeh [21]: $\mu \mathrm{A} \cap \mathrm{B}=\min [\mu \mathrm{A}(\mathrm{xi}), \mu \mathrm{B}(\mathrm{xi})]$.

The proposed fuzzy system uses the Mamdani model [26], i.e., for all rules which the relevance degree of the function is greater than zero, they will contribute to the calculation of the corresponding output of the inference system.

The membership degree resulting from the rules will limit the values of output fuzzy sets generated by these rules in accordance with the variables, i.e., the values of the operations made in the rules will characterize the resulting linguistic variable.

Afterwards, a function of global union is applied that will form the output for each output linguistic variable, i.e., for each linguistic variables present in the FLC function is performed a union operation of the respective values of the rules.

This process aims to transform the input linguistic variables into other linguistic variables corresponding to the output membership function (the FLC function). These variables, on the other hand, will be converted into a crisp value in the defuzzification process.

\subsection{Defuzzification}

In the defuzzification process of the proposed fuzzy system, the Weight Average Maximum was used as a defuzzification method, because it is a low-processing method and is within the proposal scope which considers a network formed of routers with low memory and limited processing capacity. This method produces a numerical value considering the weighted average of the central activated values where the weights are the membership degrees of each output linguistic variable.

The defuzzification function is as follows:

$$
\left[\left(1 * \mu_{H}(x)\right)+\left(2 * \mu_{M}(x)\right)+\left(4 * \mu_{L}(x)\right)\right] /\left(\mu_{H}(x)+\mu_{M}(x)+\mu_{L}(x)\right)
$$


Where $\mu \mathrm{H}(\mathrm{x})$ is the membership degree of the variable High, $\mu \mathrm{M}(\mathrm{x})$ is the membership degree of the variable Medium, and $\mu \mathrm{L}(\mathrm{x})$ is the membership degree of the variable Low. Values 1,2 , and 4 are the maximum values of the variables High, Medium, and Low, respectively as shown in Figure 3.

\section{Performance Evaluation}

This section presents the behavior and performance of the OLSR-FLC protocol in a simulation environment. The goal is to analyse and show the benefits of the proposed solution by comparing it with the main extensions of the OLSR protocol considered for this work.

We analyzed the performance of the proposal through simulations on Network Simulator (NS-2) [27], using the scenario shown in Figure 4 which represents the WMN backbone deployed at the Federal University of Para (UFPA) campus.

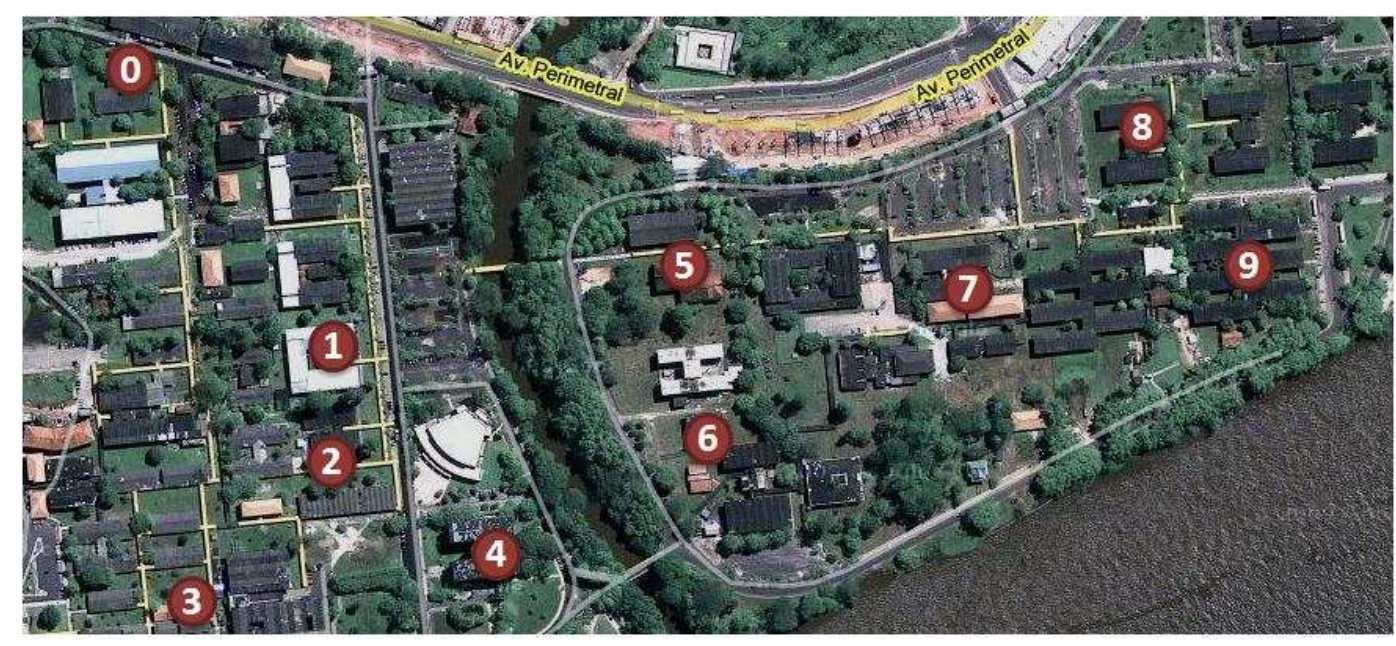

Figure 4: Considered scenario.

The simulations present the impact of the protocols on the quality of real video sequences by assessing not only the traffic from the perspective of the network (QoS paramenters), but also from the user's perspective (QoE parameters).

Table 2 shows the simulation parameters which tries to bring the simulation as close as possible to the considered network, representing the characteristics of the region and the used equipments. Path Loss Exponent and 
Shadowing Deviation parameters were used according to the measurements presented in [18]. The routers' carrier sense threshold and transmit power parameters were based on the IEEE 802.11 standard [29]. The other values were used to represent the antennas and the routers used in the WMN at UFPA.

Table 2: Simulation parameters

\begin{tabular}{|c|c|}
\hline Parameter & Value \\
\hline Standard IEEE & $802.11 \mathrm{~g}$ \\
\hline Propagation Model & Shadowing \\
\hline Antenna & Omnidirectional 18dB \\
\hline Router's Carrier Sense Threshold & $-76 \mathrm{dBm}$ \\
\hline Router's Transmit Power & $-80 \mathrm{dBm}$ \\
\hline Transmission Power & $17 \mathrm{dBm}$ (WRT54G) \\
\hline Frequency & $2.422 \mathrm{GHz}($ Channel 3$)$ \\
\hline Path Loss Exponent & 1.59 \\
\hline Shadowing Deviation & $5.4 \mathrm{~dB}$ \\
\hline
\end{tabular}

Twenty simulations were performed using different generator seeds for each protocol: OLSR, OLSR-ETX, OLSR-MD, OLSR-DC, and OLSR-FLC. Table 3 shows the flow configuration used in the simulations. All simulations were run for 50 seconds. The results in the graphs were calculated with a confidence interval of $99 \%$, according to [28].

The configuration of flows aims to balance the flows over the topology and to create a higher competition scenario, between data, audio, and video traffics. Hence, it brings the simulation to a common situation in WMNs, i.e., competition among all kind of flows where each flow has its own characteristics and requirements.

The simulation comprised 3 VoIP (Voice over IP) calls which are represented by two flows in NS-2, i.e., 6 UDP flows. Moreover, 5 TCP-Reno flows and 3 real video sequences are used. The video flows were evaluated considered the experience that the user obtained through the QoE objective and subjective metrics.

The UDP flows have a bit rate of $8 \mathrm{~Kb} / \mathrm{s}$ and 40 bytes $(\mathrm{RTP}+\mathrm{UDP}+$ Payload) of packet size in order to represent the G.729 codec [30]. The TCP flows were characterized as FTP applications following the Pareto model with a rate of 200k, 210 bytes of packet size and $500 \mathrm{~ms}$ burst duration. 
Table 3: Flow configuration

\begin{tabular}{|c|c|c|c|c|l|}
\hline Flow & Source & Destination & Begin & End & Traffic \\
\hline 1 & 1 & 8 & 10 & 40 & TCP - Reno \\
\hline 2 & 9 & 2 & 11 & 41 & TCP - Reno \\
\hline 3 & 7 & 4 & 12 & 42 & TCP - Reno \\
\hline 4 & 5 & 0 & 13 & 43 & TCP - Reno \\
\hline 5 & 6 & 4 & 14 & 44 & TCP - Reno \\
\hline 6 & 0 & 5 & 10 & 45 & Video Paris \\
\hline 7 & 3 & 6 & 14 & 29 & Video Foreman \\
\hline 8 & 3 & 6 & 30 & 45 & Video News \\
\hline 9 & 2 & 9 & 6 & 46 & UDP - CBR \\
\hline 10 & 9 & 2 & 6 & 46 & UDP - CBR \\
\hline 11 & 1 & 8 & 7 & 47 & UDP - CBR \\
\hline 12 & 8 & 1 & 7 & 47 & UDP - CBR \\
\hline 13 & 4 & 7 & 8 & 48 & UDP - CBR \\
\hline 14 & 7 & 4 & 8 & 48 & UDP - CBR \\
\hline
\end{tabular}

The video flows were simulated through the Evalvid tool [31] that allows the control of the video quality in a simulation environment. Real video sequences were used, namely "Paris", "Foreman" and "News" [32]. These videos have frames in YUV format which are compressed by MPEG-4 codec and sent at a rate of 30 frames/s. Each frame was fragmented into blocks of 1024 bytes where the packet had size of 1052 bytes.

For the sake of simplicity, the result analysis is divided into two subsections: analysis from the network's perspective and from the user's perspective.

\subsection{Network's Perspective}

The throughput graphs were divided into VoIP calls, TCP flows, and the video traffic to provide a better content view. The graphs of delay and jitter were used only to show the data concerning VoIP and video traffic as these metrics are the less important ones for the TCP flows.

Figure 5 shows the data regarding the blocking probability of each flow. The blocking probability represents the packets sent that were not delivered successfully due to bit errors and queue size, for example. The TCP flows are from 1 to 5, the video flows from 6 to 8 and the UDP flows from 9 to 14 . 


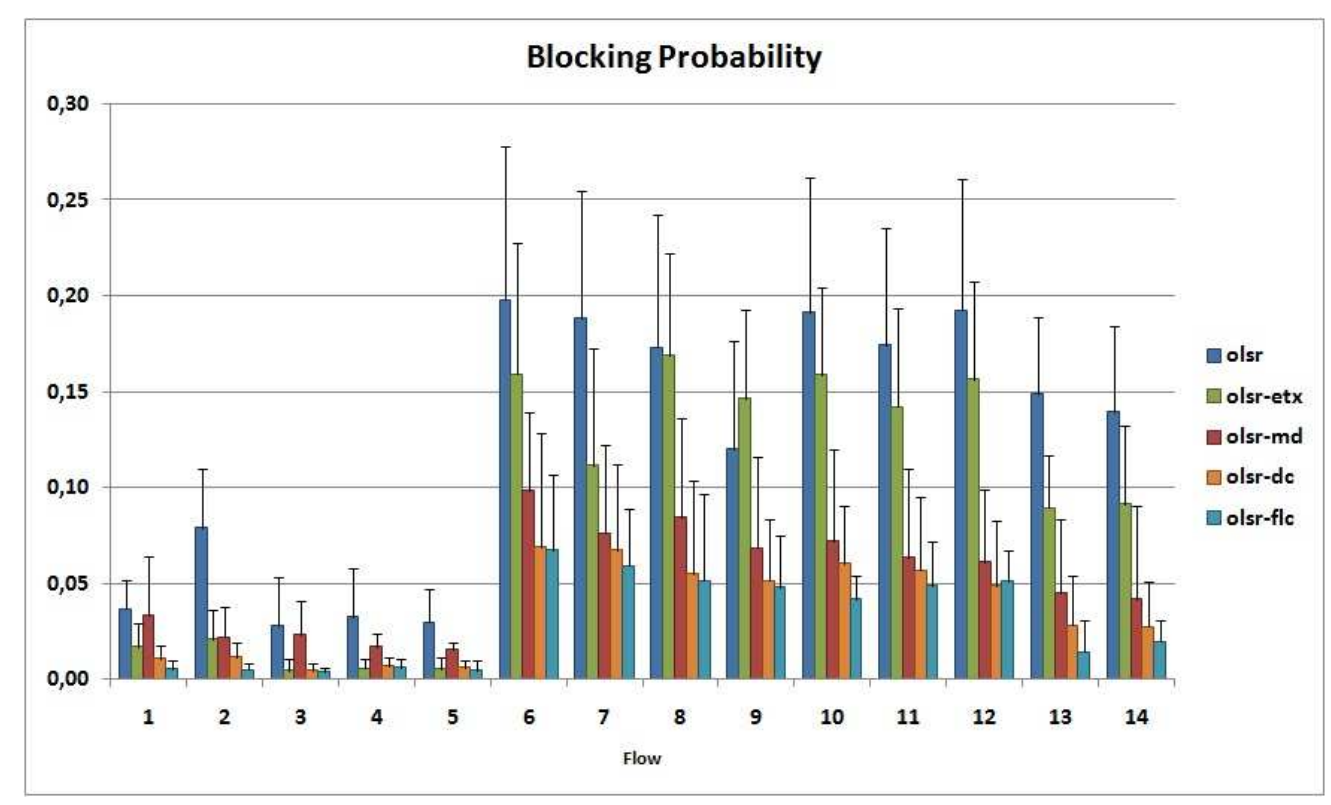

Figure 5: Blocking probability.

Based on Figure 5, it is observed that among the protocols analyzed, the protocols OLSR-DC and OLSR-FLC had the lowest values of blocking probability. This occurs due to both protocols distribute traffic more adequately through the treatment differentiation for each type of traffic. However, the OLSR-FLC protocol is more efficient because it takes into account information from more than one metric to select the best path.

When comparing the protocols individually regarding the sum of all losses occurred (i.e., the sum of all discarded packets), it is observed that OLSRFLC protocol improved the system performance in 130\% compared to OLSR protocol, and in $7.8 \%$ compared to OLSR-DC protocol that achieved the second best performance.

Figure 6 shows the throughput obtained by each protocol regarding the TCP flows, Figure 7 presents the system behavior for the VoIP calls, and Figure 8 illustrates the video transmission data.

The results show that the OLSR-DC and OLSR-FLC protocols had the best throughput performance for flows exchanged between nodes placed far way, flows 1 and 2 in Figure 6, while the protocol OLSR-ETX obtained better results for flows between nodes that are closer. This occured due to the time to differentiate TCP and UDP packets which generated an overhead for the 


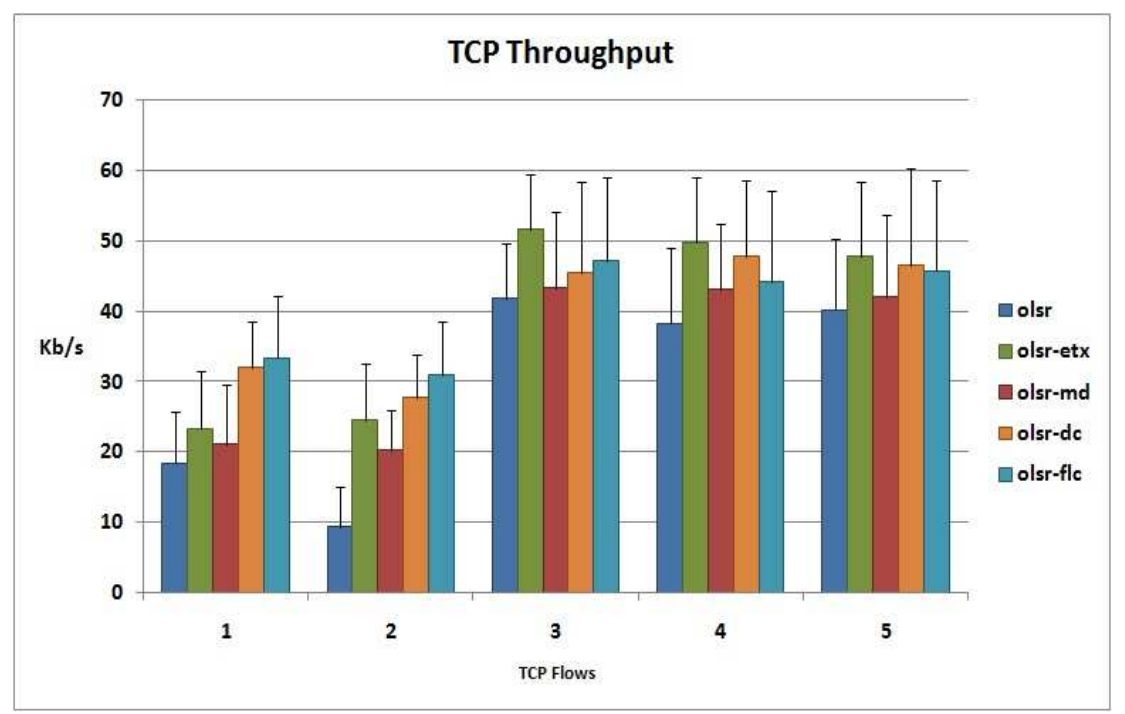

Figure 6: TCP throughput.

OLSR-DC and OLSR-FLC protocols.

Figure 7 shows that the OLSR-FLC protocol had the best performance among the protocols followed by the OLSR-DC protocol. The results show that the traffic differentiation becomes advantageous especially for flows spanning great distances. The distance factor brings a need to use more hops, making the path choice more important. Although both protocols provide traffic differentiation, the higher performance of OLSR-FLC protocol occured due to the utilization of more than one metric (ETX and MD) to generate the fuzzy link cost used for routing.

The close performance of the OLSR-MD, OLSR-DC and OLSR-FLC protocols occured because video traffics are between nodes relatively close. This short distance, when compared to other flows, makes the number of hops smaller. Therefore, it reduces the importance of having to choose the best node to route packets since the possibility of direct communication is the best option approximating the performance of the protocols.

Regarding flows 7 and 8, the nodes involved in this communication have a greater distance when compared to flow 6 , and they also have other nodes that prevent a clear line of sight between them, causing higher interference. Although these flows are between the same nodes, they begin at different times, as shown in Table 3. Flow 7 begins at a time of extreme competition between the flows in the network. Therefore, it has more difficulty for data 


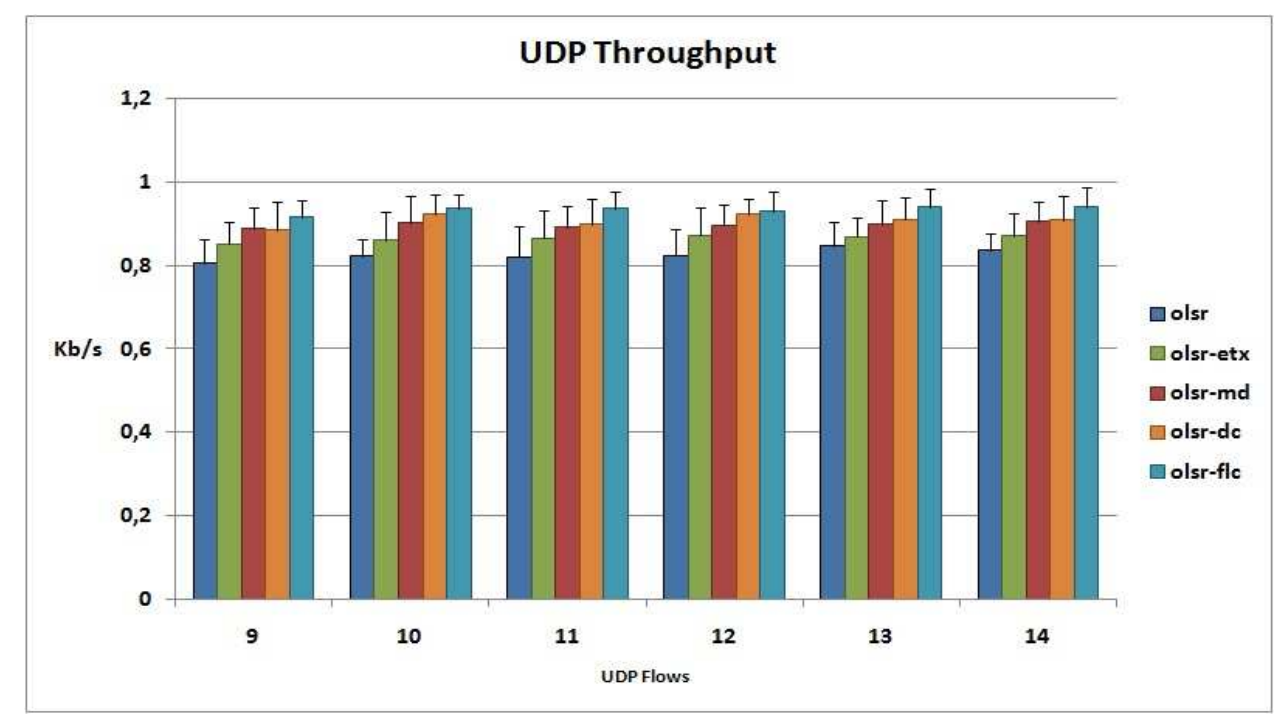

Figure 7: UDP throughput.

transmission, which does not occur with flow 8.

Figure 9 depicts the delay of VoIP calls and video transmissions. Flow 6, despite having higher throughput than the other video transmissions as shown in Figure 8, it also had a higher delay due to the use of other nodes as hops along the communication path.

When using other nodes as hops to overcome the distances and congestion, other types of delay appear, such as the propagation delay and the queue/error checking delay, increasing the end-to-end delay. The competition scenario can generate routes with high numbers of hops to use network resources more efficiently.

Regarding other flows, note that OLSR-FLC protocol can achieve the best delay performance, by using a link cost which represents the link quality (ETX) and also takes into account the link delay (MD).

Figure 10 presents the jitter results for VoIP calls and video transmissions. Despite having a high value of delay on flow 6, as shown in Figure 9, OLSRFLC had a low jitter behavior compared to other protocols, and the same occured with the other transmissions shown. This occured because OLSRFLC uses a link cost based on a fuzzy system. This characteristic changes the routes only when the new route discovery has values much better than the current route which results in a small number of route change.

This less frequent route change makes packets follow a specific route more 


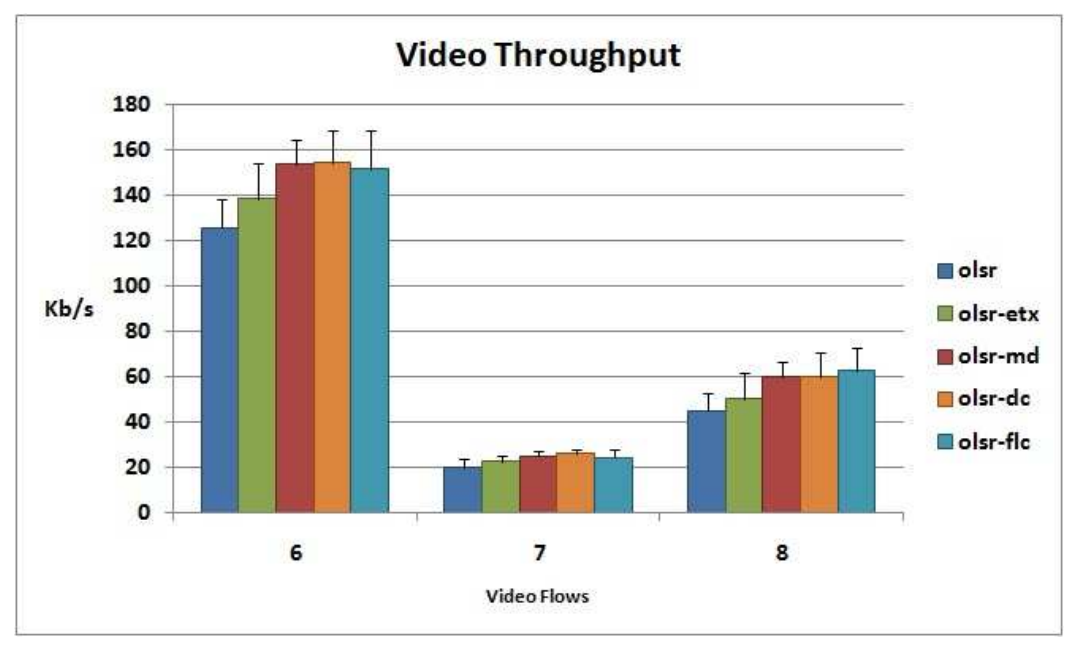

Figure 8: Video throughput.

constantly, avoiding out-of-order delivery of theses packets which can increase jitter for a flow if it happens otherwise.

\subsection{User's Perspective}

We used objective and subjective QoE metrics to verify the quality evaluation of the video transmissions following the experiments proposed in [33]. The well-known subjective QoE metric, Mean Opnion Score (MOS), was used to assess the quality of multimedia applications based on the user's opinion.

MOS is a scale for assessing perceptions of the video that attempts to quantify the quality of the video based on the human perception. The user qualifies the video according to the experience obtained with it. This qualification is done through a "grade" which is given to the video by the user [34].

Objective metrics for QoE estimate the quality of received video through quantitative mathematical models whose computed values are mapped into subjective values of quality. The main objective metrics are: Peak Signal to Noise Ratio (PSNR), Structural Similarity (SSIM), and Video Quality Metric (VQM) [34].

The PSNR is the most traditional objective metric and compares frame by frame the quality of the video received by the user with the original one [35]. The SSIM is a measurement of the video structural distortion trying to get a better correlation with the user's subjective impression where values 


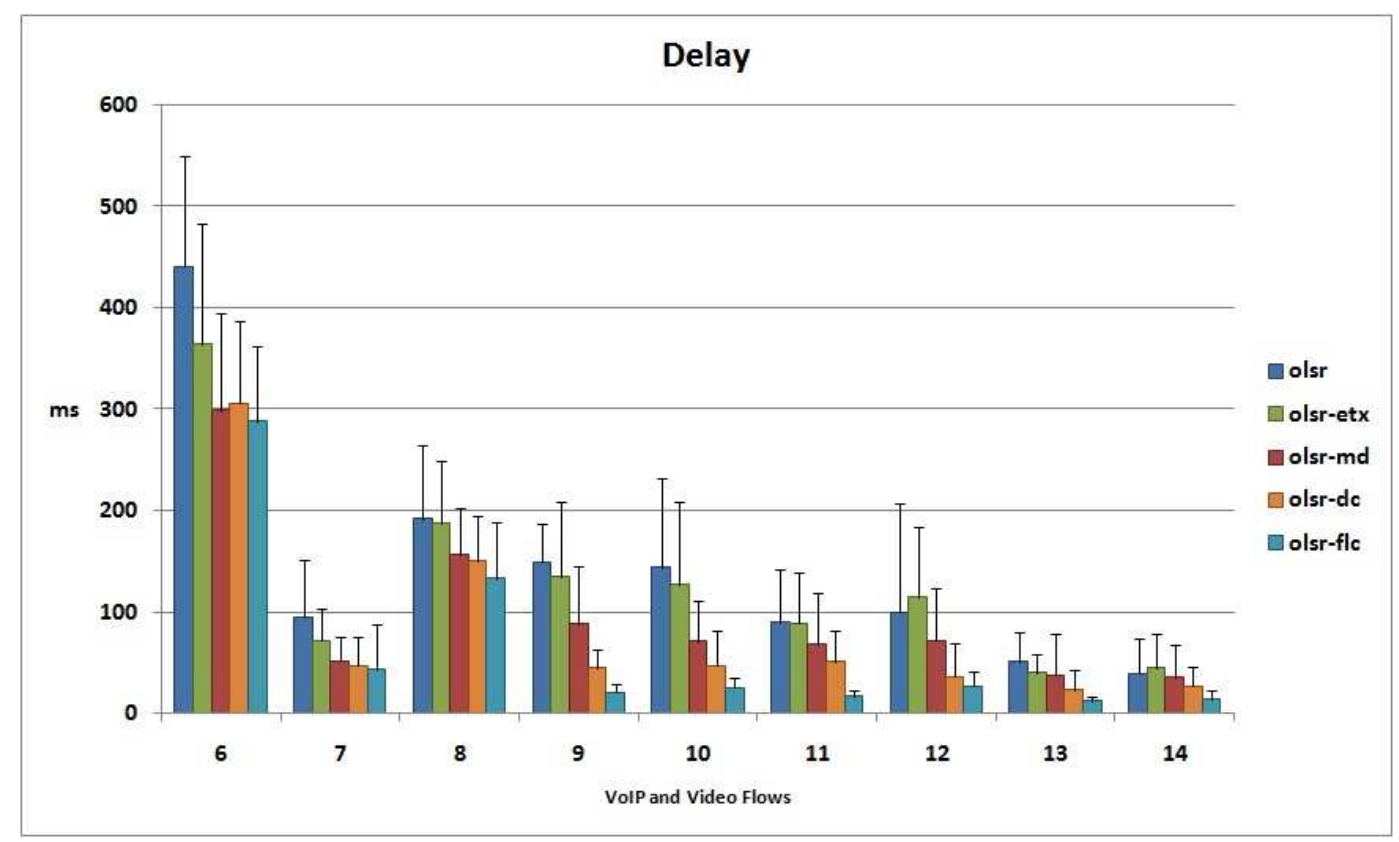

Figure 9: Delays per flow.

vary between 0 and 1 . The closer the metric gets to 1 , the better the video quality [36] [37].

The VQM metric measures the perception damage the video experienced based on Human Visual System (HVS) characteristics including in a single metric factors such as blurring, noise, color distortion, and distortion blocks. VQM gets values between 0 and 5, where 0 is the best quality [38].

The videos were analyzed using the MSU Video Quality Measurement Tool Software [39]. The value of PSNR is expressed in dB (decibels). For a video to be considered with good quality, it should have an average PSNR of at least $30 \mathrm{~dB}$. This is based on the mapping of PSNR values to MOS shown in Table 4 [34].

Next, it is presented the tables containing information of QoE metrics regarding each video collected from all protocols. The tables show the average, the highest, and the lowest values as well as the standard deviation for each protocol. 


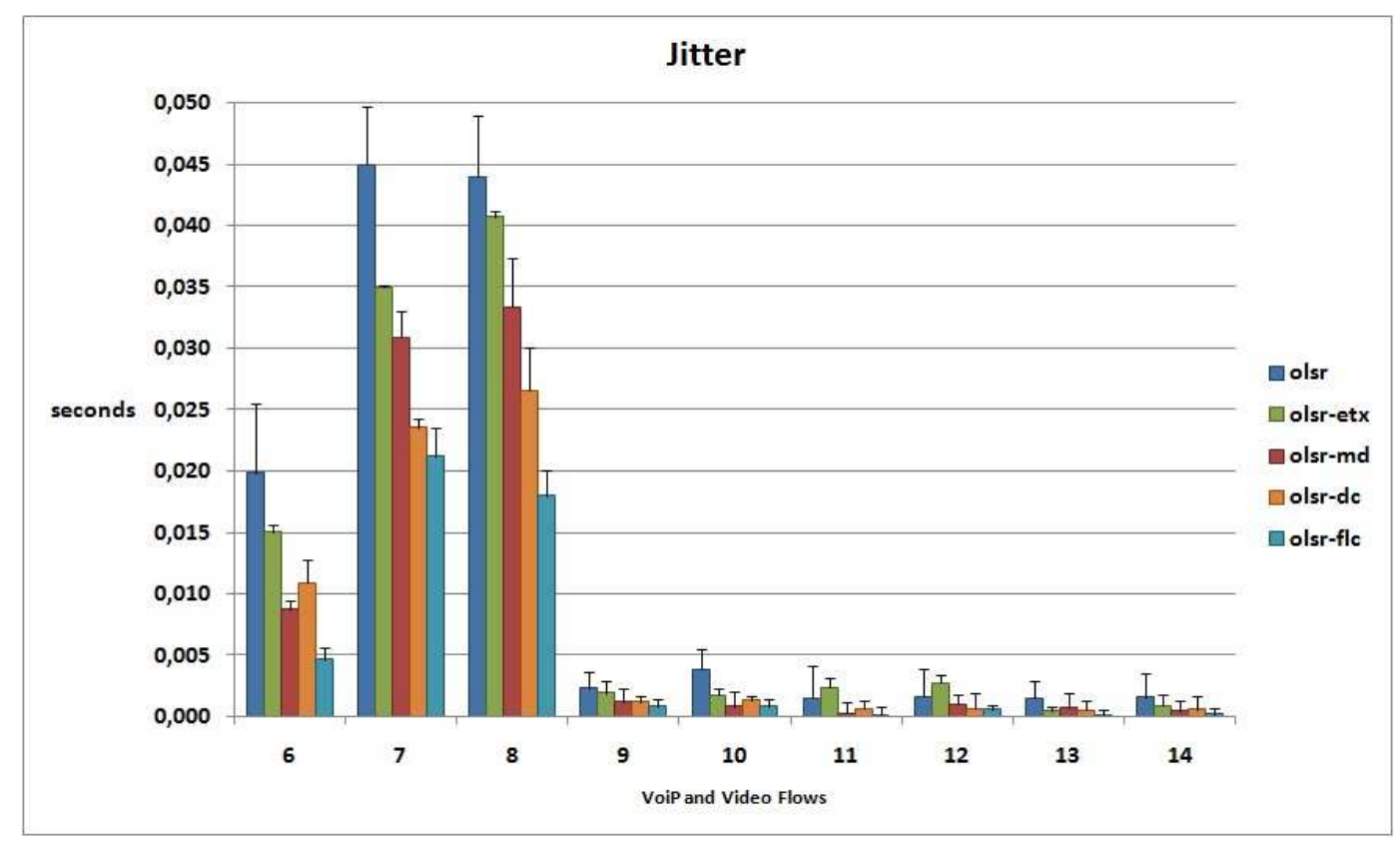

Figure 10: Jitter per flow.

\subsubsection{Foreman}

Tables 5 to 7 show the values for the video "Foreman", where Table 5 presents the values of VQM metric, Table 6 the SSIM values, and Table 7 the values of PSNR and MOS metrics.

The transmission of the "Foreman" video, flow 7, begins after all flows start their transmissions, and it starts in a moment of convergence of the protocols which results in a very difficult transmission due to network congestions. These facts become clear from the data shown in the tables for the "Foreman" Video. However, OLSR-FLC had the best performance for the QoE metrics, and is the only one which achieved the "Poor" quality while the other protocols obtained a quality considered "Bad".

\subsubsection{News}

Tables 8 to 10 show the values for the video "News", where Table 8 presents the values of VQM metric, Table 9 the SSIM values, and Table 10 the values of PSNR and MOS metrics.

The "News" video, flow 8, has the same destination and source as flow 7 , "Foreman" video. However, it starts at a different time of the simulation. At 
Table 4: PSNR / MOS Mapping.

\begin{tabular}{|c|c|}
\hline PSNR (dB) & MOS \\
\hline$>37$ & (5) Excellent \\
\hline $31-37$ & $(4)$ Good \\
\hline $25-30$ & (3) Regular \\
\hline $20-25$ & $(2)$ Poor \\
\hline$<20$ & $(1)$ Bad \\
\hline
\end{tabular}

Table 5: VQM Values of Video Foreman

\begin{tabular}{|c|c|c|c|c|}
\hline Foreman & & & VQM & \\
\hline & Higher & Lower & Average & Deviation \\
\hline OLSR & 5 & 4,8 & 4,96 & 0,07 \\
\hline OLSR-MD & 5 & 4 & 4,72 & 0,32 \\
\hline OLSR-ETX & 5 & 4,5 & 4,86 & 0,23 \\
\hline OLSR-DC & 5 & 2,3 & 4,50 & 0,83 \\
\hline OLSR-FLC & 4,8 & 2,4 & 4,27 & 0,71 \\
\hline
\end{tabular}

this moment, the protocols had already converged allowing a better choice of routes. We noted this by comparing the performance of both videos where the "News" video had better results for QoE metrics.

OLSR-FLC achieved the best video quality having a quality considered "Regular", while the other protocols obtained qualities ranging from "Poor" to "Bad". Despite having a better video quality rating, OLSR-FLC had a high standard deviation showing a degree of instability in the quality of the transmitted videos, obtaining values better and of similar quality as other protocols.

\subsubsection{Paris}

Tables 11 to 13 show the values for the video "Paris", where Table 11 presents the values of VQM metric, Table 12 the SSIM values and Table 13 the values of PSNR and MOS.

Since the "Paris" video, flow 6, is longer than the other videos, it is transmitted during almost the entire simulation. This means that the flow had a hard time during the convergence of the protocols at the beginning of its transmission, but most of the communication occurs after the convergence period. 
Table 6: SSIM Values of Video Foreman

\begin{tabular}{|c|c|c|c|c|}
\hline Foreman & & & SSIM & \\
\hline & Higher & Lower & Average & Deviation \\
\hline OLSR & 0,70 & 0,50 & 0,58 & 0,07 \\
\hline OLSR-MD & 0,71 & 0,62 & 0,66 & 0,04 \\
\hline OLSR-ETX & 0,77 & 0,50 & 0,61 & 0,08 \\
\hline OLSR-DC & 0,87 & 0,61 & 0,67 & 0,08 \\
\hline OLSR-FLC & 0,83 & 0,68 & 0,73 & 0,04 \\
\hline
\end{tabular}

Table 7: PSNR and MOS Values of Video Foreman

\begin{tabular}{|c|c|c|c|c|c|}
\hline Foreman & & PSNR & & MOS \\
\hline & Higher & Lower & Average & Deviation & \\
\hline OLSR & 18 & 14 & 15,80 & 1,62 & Bad \\
\hline OLSR-MD & 23 & 16 & 19,10 & 2,26 & Bad \\
\hline OLSR-ETX & 20 & 13 & 17,40 & 2,63 & Bad \\
\hline OLSR-DC & 25 & 17 & 19,30 & 2,58 & Bad \\
\hline OLSR-FLC & 25 & 22 & 22,90 & 0,88 & Poor \\
\hline
\end{tabular}

Unlike the other video transmissions, flows 7 and 8, the nodes involved in flow 6 have a clear line of sight, however, with a greater distance between the nodes. This makes that the use of a single hop increases the chance of packet losses as well as the use of multiples hops increases the end-to-end delay of the packets.

Within this reality, the usage of a single metric turns out to be insufficient to find the most appropriate route, because a good video transmission depends not only on small losses, but also on a small delay and jitter.

Therefore, we observed that OLSR-FLC can adapt to this reality of multiple requirements, which is visible in the tables for the "Paris" video. The OLSR-FLC protocol, as well as the OLSR-DC protocol, had a video quality considered "regular", however OLSR-FLC reaches values close to "Good" quality level.

In other words, since it is based on the OLSR-DC protocol, the OLSRFLC protocol can better distribute the traffic, but it uses a fuzzy link cost based on delay and quality of links. This enables the protocol to obtain a better video quality, against the protocols that use only one metric for routing. 
Table 8: VQM Values of Video News.

\begin{tabular}{|c|c|c|c|c|}
\hline News & & & VQM & \\
\hline & Higher & Lower & Average & Deviation \\
\hline OLSR & 5 & 1,7 & 4,43 & 1,08 \\
\hline OLSR-MD & 5 & 2,2 & 3,71 & 1,05 \\
\hline OLSR-ETX & 5 & 2,2 & 4,09 & 1,01 \\
\hline OLSR-DC & 4,9 & 1,4 & 3,44 & 1,17 \\
\hline OLSR-FLC & 4 & 0,5 & 3,08 & 1,01 \\
\hline
\end{tabular}

Table 9: SSIM Values of Video News

\begin{tabular}{|c|c|c|c|c|}
\hline News & & & SSIM & \\
\hline & Higher & Lower & Average & Deviation \\
\hline OLSR & 0,86 & 0,71 & 0,79 & 0,05 \\
\hline OLSR-MD & 0,95 & 0,83 & 0,89 & 0,04 \\
\hline OLSR-ETX & 0,89 & 0,78 & 0,85 & 0,04 \\
\hline OLSR-DC & 0,97 & 0,84 & 0,89 & 0,05 \\
\hline OLSR-FLC & 0,98 & 0,85 & 0,91 & 0,04 \\
\hline
\end{tabular}

This evaluation shows that only one metric for routing data and multimedia traffics may not be sufficient to reach acceptable QoS and QoE levels to answer the needs of such traffics since each of them has different requirements.

Therefore, we have shown that from the moment that each traffic is treated according to its needs (traffic differentiation in routing) and more than one metric is considered to answer such needs, the routing protocol gets closer to the ideal requirements of each type of traffic.

\section{Conclusion and Future Work}

This work presented an extended version of the OLSR protocol, OLSRFLC (Fuzzy Link Cost), that has been developed based on an existing version called OLSR-DC protocol. This new version uses fuzzy logic to build a fuzzy system that aims to solve the problem of using multiple metrics for routing and increases the multimedia experience.

The proposed fuzzy system has as basis the values of the ETX and MD metrics collected from the network to define the FLC, which is then used to 
Table 10: PSNR and MOS Values of Video News

\begin{tabular}{|c|c|c|c|c|c|}
\hline News & & PSNR & & & MOS \\
\hline & Higher & Lower & Average & Deviation & \\
\hline OLSR & 25 & 17 & 19,70 & 2,91 & Bad \\
\hline OLSR-MD & 27 & 19 & 22,50 & 3,21 & Poor \\
\hline OLSR-ETX & 29 & 17 & 20,70 & 4,22 & Poor \\
\hline OLSR-DC & 27 & 19 & 23,60 & 2,84 & Poor \\
\hline OLSR-FLC & 44 & 20 & 25,70 & 7,01 & Regular \\
\hline
\end{tabular}

Table 11: VQM Values of Video Paris

\begin{tabular}{|c|c|c|c|c|}
\hline Paris & & & VQM & \\
\hline & Higher & Lower & Average & Deviation \\
\hline OLSR & 5 & 4,4 & 4,93 & 0,19 \\
\hline OLSR-MD & 3,8 & 3 & 3,35 & 0,31 \\
\hline OLSR-ETX & 4,9 & 3 & 4,09 & 0,59 \\
\hline OLSR-DC & 3,5 & 2,3 & 2,94 & 0,41 \\
\hline OLSR-FLC & 3,1 & 2,3 & 2,75 & 0,32 \\
\hline
\end{tabular}

route packets. TCP packets are still routed based on the ETX metric, as occurs in the OLSR-DC protocol.

The results show that the performance of the OLSR-FLC protocol was superior when compared to other OLSR versions reaching up to $130 \%$ improvement in system performance. The best performance of the OLSR-FLC protocol occurs not only from the network's perspective, but also from the user's perspective according to the evaluated QoE metrics.

As future work, we intend to develop and integrate other QoE metrics and video characteristics into the proposed fuzzy system, and evaluate new models of existing fuzzy systems in a simulated and experimental environment.

\section{Acknowledgements}

The authors thank the financial support of CNPq, project: 557.128/20099 (Brazilian Institute of Web Science Research). 
Table 12: SSIM Values of Video Paris

\begin{tabular}{|c|c|c|c|c|}
\hline Paris & & & SSIM & \\
\hline & Higher & Lower & Average & Deviation \\
\hline OLSR & 0,75 & 0,63 & 0,69 & 0,04 \\
\hline OLSR-MD & 0,90 & 0,83 & 0,86 & 0,02 \\
\hline OLSR-ETX & 0,83 & 0,73 & 0,79 & 0,04 \\
\hline OLSR-DC & 0,93 & 0,87 & 0,88 & 0,02 \\
\hline OLSR-FLC & 0,93 & 0,87 & 0,91 & 0,02 \\
\hline
\end{tabular}

Table 13: PSNR and MOS Values of Video Paris

\begin{tabular}{|c|c|c|c|c|c|}
\hline Paris & & PSNR & & & MOS \\
\hline & Higher & Lower & Average & Deviation & \\
\hline OLSR & 17 & 14 & 15,20 & 1,23 & Bad \\
\hline OLSR-MD & 27 & 23 & 24,80 & 1,14 & Poor \\
\hline OLSR-ETX & 23 & 20 & 21,40 & 1,17 & Poor \\
\hline OLSR-DC & 29 & 23 & 26,50 & 2,07 & Regular \\
\hline OLSR-FLC & 31 & 25 & 29,20 & 2,15 & Regular \\
\hline
\end{tabular}

\section{References}

[1] Y. Zhang, J. Luo and H. Hu. Wireless mesh networking: Architectures, protocols and standards. Auerbach Publications, 2006.

[2] Y. Li, Z. Li, M. Chiang, A. R. Calderbank. Content-Aware DistortionFair Video Streaming in Congested Networks. Multimedia, IEEE Transactions on , 11(6), 1182-1193, Oct. 2009.

[3] B. Ciubotaru, Muntean G. M.. Quality of multimedia streamingoriented handover management solution for mobile applications. $B M S B$ '09. Broadband Multimedia Systems and Broadcasting, IEEE International Symposium on. 1-5, May 2009.

[4] J. Okamoto, K. Watanabe, A. Honda, M. Uchida, S. Hangai. HDTV objective video quality assessment method applying fuzzy measure. Quality of Multimedia Experience, 2009. QoMEx 2009. International Workshop on. 168-173, July 2009.

[5] D. M. Ngoc, P. A. Tan, J. S. Lee, H. Oh. Improvement of IEEE 802.11 
for multimedia traffic in wireless LAN. Wireless Telecommunications Symposium, 200\%. WTS 200\%. 1-4, April, 2007.

[6] M. Mu, E. Cerqueira, F. Boavida, A. Mauthe. Quality of Experience management framework for real-time multimedia applications. Int. J. Internet Protoc. Technol. 4(1), Inderscience Publishers, 54-64, 2009.

[7] Borges, V. C., Pereira, D., Curado, M., and Monteiro, E. Routing Metric for Interference and Channel Diversity in Multi-Radio Wireless Mesh Networks. ADHOC-NOW '09: Proceedings of the 8th International Conference on Ad-Hoc, Mobile and Wireless Networks, SpringerVerlag, 55-68, 2009.

[8] S. Lekcharoen, C. Chaochanchaikul, C. Jittawiriyanukoon. A design fuzzy control policing mechanisms on quality of service support in wireless networks. Proceedings of the 3rd international conference on Mobile technology, applications and systems, Oct. 2006.

[9] A. Takahashi, D. Hands and V. Barriac. Standardization Activities in the IUT for a QoE Assessment of IPTV. IEEE Communication Magazine, Vol. 46, Issue 2, 2008.

[10] ITU-T. International Telecommunication Union - Telecommunication Standardization Sector. Available at http://www.itu.int/ITU-T/, Accessed in September of 2009.

[11] VQEG. Video Quality Experts Group. Available at http://www.its.bldrdoc.gov/vqeg/, Accessed in September of 2009.

[12] ETSI STQ. European Technical Committee for Speech, Transmission, Planning, and Quality of Service. Available at http://portal.etsi.org, Accessed in September of 2009.

[13] T. Clausen and P. Jacquet. Optimized link state routing protocol (OLSR) - RFC 3626. http://www.ietf.org/rfc/rfc3626.txt, 2006.

[14] D. De Couto, D. Aguayo, J. Bicket and R. Morris. A high-throughput path metric for multi-hop wireless routing. 9th Annual International Conference on Mobile Computing and Networking, pages 134-146, 2003. 
[15] W. Cordeiro, E. Aguiar, W. Moreira, A. Abelem and M. Stanton. Providing quality of service for mesh networks using link delay measurements. 16th International Conference on Computer Communications and Networks, pages $991-996,2007$.

[16] R. Gomes, W. Moreira, V. Nascimento and A. Abelem. Dynamic metric choice routing for mesh networks. 7th International Information and Telecommunication Technologies Symposium (IRTS), 2008.

[17] Z. Wang, J. Crowcroft. Quality-of-service routing for supporting multimedia applications. Selected Areas in Communications, IEEE Journal on, vol. 14, no. 7, pp. 1228-1234, September. 1996.

[18] W. Moreira, E. Aguiar, A. Abelém and M. Stanton. Using multiple metrics with the optimized link state routing protocol for wireless mesh networks. $26^{\circ}$ Simpósio Brasileiro de Redes de Computadores e Sistemas Distribuídos, Maio 2008.

[19] R. Jain. Quality of experience. IEEE Multimidia, 11:90-98, June 2004.

[20] Z. Wang, A. Bovik and L. Lu. Why is image quality assessment so difficult? IEEE International Conference on Acoustics, Speech and Signal Processing, May 2002.

[21] L. A. Zadeh. Fuzzy Sets. Information and Control, Vol.8, 1965.

[22] H. Adeli, K. C. Sarma. Cost Optimization of Structures: Fuzzy Logic, Genetic Algorithms, and Parallel Computing. Wiley, 2006.

[23] R. Zhang, K. Long. A fuzzy routing mechanism in next-generation networks. Proc. IASTED International Conference on Intelligent Systems and Control (ISC). Oct. 2002.

[24] E. Aboelela, C. Douligeris. Routing in multimetric networks using a fuzzy link cost. Proceedings of the 2nd IEEE Symposium on Computers and Communications (ISCC' '97). 1997.

[25] S. Ghosh, Q. Razouqi, H. J. Schumacher, A. Celmins. A survey of recent advances in fuzzy logic in telecommunications networks and new challenges. IEEE Trans. Fuzzy Systems. 1998. 
[26] D.H. Anderson, L.O. Hall. MR. FIS: Mamdani rule style fuzzy inference system. IEEE International Conference on Systems, Man, and Cybernetics 1999.

[27] K. Fall and K. Varadhan. The network simulator - ns-2. http://www.isi.edu/nsnam/ns/.

[28] R. Jain. The Art of Computer Systems Performance Analysis: Techniques for Experimental Design, Measurement, Simulation, and Modeling. Wiley- Interscience, New York, NY, April 1991. ISBN:0471503361.

[29] Institute of Electrical and Electronic Engineering. IEEE 802.11, 1999 Edition (ISO/IEC 8802-11: 1999): Information Technology - Telecommunications and Information Exchange between Systems - Local and Metropolitan Area Network. 1999.

[30] J. Balam and J. Gibson. Multiple descriptions and path diversity for voice communications over wireless mesh networks. IEEE Transactions on Multimedia, pages 1073-1088, August 2007.

[31] Evalvid. http://www.tkn.tu-berlin.de/research/evalvid/.

[32] Foreman, Paris and News. http://trace.eas.asu.edu/yuv/index.html.

[33] ITU - R Recommendation BT500-7. Methodology for the subjective assessment of the quality of television pictures. Technical Report, 1990.

[34] C. Lambrecht and O. Verscheure. Perceptual quality measure using a spatio - temporal model of the human visual system. Digital Video Compression: Algorithms and Technologies, pages 450-461, 1996.

[35] Z. Wang, L. Lu and A. Bovick. Video quality assessment based on structural distortion measurement. Signal Processing: Image Communication, special issue on "Objective Video Quality Metrics, 2004.

[36] Z. Wang and A. Bovik. A universal image quality index. IEEE Signal Processing Letters, pages 81-84, 2002.

[37] Z. Wang and A. Bovik. Image quality assessment from error visibility to structural similarity. IEEE Trans Image Processing, 2004. 
[38] M. Pinson and S. Wolf. A new standardized method for objectively measuring video quality. IEEE Transacions on Broadcasting, 2004.

[39] MSU Video Quality Measurement Tool Software. http://compression.ru/video/quality_measure/index_en.html. 